\title{
Not so sweet-artificial sweeteners can cause glucose intolerance by affecting the gut microbiota
}

N oncaloric artificial sweeteners (NAS) have been linked with glucose intolerance in a new study published in Nature. Furthermore, the researchers have demonstrated that the metabolic changes underlying glucose intolerance are the result of consumed NAS altering the composition of the gut microbiota.

The incidence of the metabolic syndrome has increased to epidemic proportions over the past few decades, which is probably linked to changes in human nutrition. A notable change over this period is the increased use of NAS in common foods, in an effort to reduce caloric intake and normalize blood levels of glucose.

However, "the safety and efficacy of NAS use remains controversial and poorly studied," explain Eran Segal and Eran Elinav, two authors of the Nature paper. Although NAS are not absorbed, they do come into contact with the gut microbiota, which is now known to have a range of effects on human physiology. The researchers therefore hypothesized that NAS might affect the composition and function of the gut microbiota, which would in turn contribute to the development of the metabolic syndrome.

To test this hypothesis, the researchers added commercial formulations of saccharin, sucralose or aspartame (commonly used NAS) to the drinking water of lean 10-week-old C57Bl/6 mice. After 11 weeks of treatment, all of the mice consuming NAS had developed glucose intolerance, whereas none of the control mice (fed water, glucose or sucrose) had. As saccharin led to the most pronounced effect, this sweetener was used in subsequent experiments. To examine the effects of NAS in obesity, C57Bl/6 mice were fed a high-fat diet with or without saccharin. Just as in the lean state, mice fed a highfat diet and saccharin developed glucose intolerance and the control mice did not.

Segal, Elinav and colleagues then went on to investigate the effect of consuming NAS on the gut microbiota. Mice were given antibiotics while they were maintained on a normal or high-fat diet supplemented with NAS. After 4 weeks of treatment with antibiotics, the mice receiving NAS and the control mice no longer had different levels of glucose intolerance, both in the lean and obese states. This result suggests that glucose intolerance induced by NAS is mediated by changes to the gut microbiota. Faecal transplantation was used to determine whether the gut microbiota had a causal role in this glucose intolerance. Germ-free mice on a normal diet received a sample either from mice fed a normal diet with saccharin or control mice. The recipients of the saccharin-related microbiota developed glucose intolerance, whereas recipients of microbiota from control mice did not, suggesting that the role of microbiota is causal.

$16 \mathrm{~S}$ ribosomal RNA gene sequencing revealed that the mice fed saccharin had a different gut microbiota composition compared with their own baseline microbiota profile and that of all the control mice. Saccharin consumption altered the abundance of $>40$ operational taxonomic units. For example, the abundance of species from the Bacteroides genus and Clostridiales order increased following saccharin consumption.

Metagenomic analyses showed that several metabolic pathways were altered in mice fed saccharin. "One such characteristic alteration was enhanced glycosaminoglycan degradation, resulting in high levels of short-chain fatty acids that are suggestive of enhanced energy harvest by the microbiota, previously reported to be linked to susceptibility to the metabolic syndrome," say Segal and Elinav.

The effect of NAS in humans was then examined. The researchers used data from an ongoing clinical nutritional study, including information on the consumption of NAS and clinical parameters of 381 participants who did not have diabetes mellitus. Consumption of NAS was positively associated with several parameters related to the metabolic syndrome, including increased weight and fasting blood levels of glucose. Similarly to the findings in mice, $16 \mathrm{~S}$ ribosomal RNA gene sequencing showed that participants who consumed NAS had a different microbiota composition compared with those who did not consume NAS, independently of BMI.

When seven healthy human volunteers who did not usually consume NAS were given saccharin for 7 days, four participants experienced poorer glycaemic responses than before they consumed NAS. When stool from these participants was transferred to germ-free mice, those that received a faecal transplant from NAS responders developed glucose intolerance, whereas mice receiving a transplant from NAS nonresponders did not.

The researchers conclude that consumption of NAS induces glucose intolerance by altering the composition and function of the gut microbiota. "Perhaps most importantly, and of potential importance for a vast number of people, our findings linking consumption of NAS, dysbiosis and the potential for glucose intolerance in some people calls for a reassessment of the massive and unsupervised use of NAS that is practiced today," conclude Segal and Elinav.

\section{Claire Greenhill}

Original article Suez, J. et al. Artificial sweeteners induce glucose intolerance by altering the gut microbiota. Nature doi:10.1038/nature13793 